\title{
Switched Systems With Multiple Invariant Sets ${ }^{\text {ts }}$
}

\author{
Michael Dorothy ${ }^{\mathrm{a}, \mathrm{b}}$, Soon-Jo Chung ${ }^{\mathrm{a}, *}$ \\ ${ }^{a}$ Department of Aerospace Engineering, University of Illinois at Urbana-Champaign, \\ Urbana, Illinois 61801 \\ ${ }^{b}$ Army Research Laboratory, Aberdeen Proving Ground, MD, 21005
}

\begin{abstract}
This paper explores dwell time constraints on switched systems with multiple, possibly disparate invariant limit sets. We show that, under suitable conditions, trajectories globally converge to a superset of the limit sets and then remain in a second, larger superset. We show the effectiveness of the dwell-time conditions by using examples of switching limit cycles commonly found in robotic locomotion and flapping flight.
\end{abstract}

Keywords: Switched systems, Non-equilibrium steady state, Set-based control

\section{Introduction}

Bifurcations have been of interest to dynamical systems theory for decades. However, most control strategies view such behavior as damaging and try to mitigate it [1]. Relatively less work actively inserts bifurcations as part of a control strategy. One example is using a classic Hopf bifurcation for modeswitching between flapping and gliding flight in micro-aerial vehicles [2]. The authors consider a supercritical Andronov-Hopf bifurcation model of $\mathbf{x}=(u ; v)$ :

$$
\dot{\mathbf{x}}=\mathbf{f}(\mathbf{x}, t ; \rho)=\left(\begin{array}{c}
-\lambda / \rho^{2}\left(u^{2}+v^{2}-\rho^{2} \sigma\right) u-\omega(t) v \\
\omega(t) u-\lambda / \rho^{2}\left(u^{2}+v^{2}-\rho^{2} \sigma\right) v
\end{array}\right)
$$

\footnotetext{
This work was supported in part by ARCS Illinois and NSF grants 1253758 \& 1427111

*Corresponding Author. Tel.: (217) 244-2737

Email addresses: michael.r.dorothy.civ@mail.mil (Michael Dorothy), sjchung@illinois.edu (Soon-Jo Chung)
} 


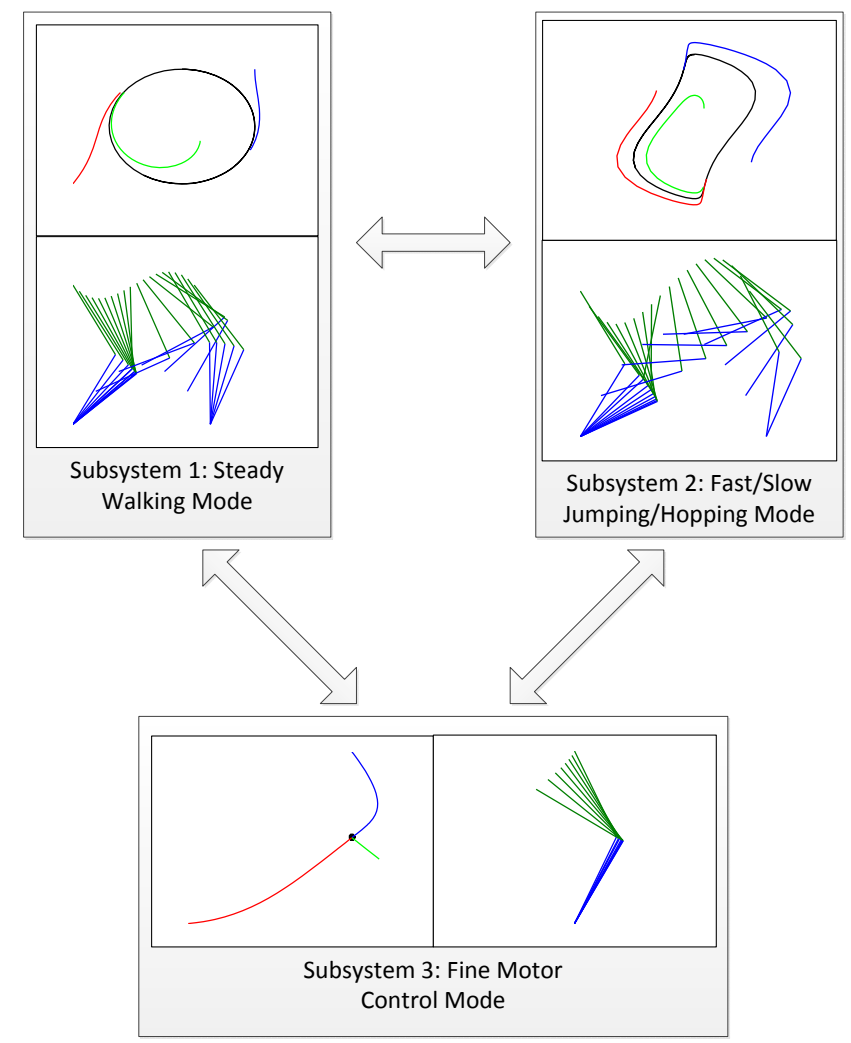

Figure 1: Schematic of mode switching with non-equilibrium limit sets.

with $\sigma=1$. For a positive rate of convergence $\lambda>0$, it can be easily shown that any initial trajectory $(u ; v) \neq \mathbf{0}$ exponentially converges to a circle of the radius $\rho$ rotating at the time-varying frequency $\omega(t)$ with bounded $\dot{\omega}(t)$. If $\sigma \leq 0$, bifurcation occurs and the system globally converges to the origin, which is useful for fast inhibition of oscillation. Fast inhibition and synchronization of oscillators are key properties for many neurobiologicallyinspired control schemes.

Another possible application is walking robots. Fig. 1 shows a hypothetical switching pattern for a walking robot application utilizing central pattern generation. A guidance/navigation engineer may design limit cycle subsystems for walking and jumping modes (shown as a Hopf oscillator and a Van der Pol oscillator), while utilizing steady-state control strategies for static balancing or tasks requiring fine motor control.

Mode-switching also implicates a large body of literature on switched 
systems [3]. Most work on stability of switched systems assumes that all subsystems have a common equilibrium point. $[4,5,6]$ consider weak Lyapunov functions in the style of LaSalle for a common equilibrium. [7] considers equilibrium location changes, but holds the vector field constant. They connect the result to averaging theory. [8] considers practical stability of affine systems with multiple distinct equilibria. Alpcan and Başar investigated dwell time criteria for nonlinear globally exponentially stable subsystems which could have differing equilibria [9]. Such systems have no single globally attractive equilibrium point. The authors of [9] reported an explicit construction of the dwell time and a conservative invariant set. This paper is inspired by that work and is a generalization of it. We generalize their result to switched systems where each subsystem may have multiple invariant sets. We pursue a similar dwell time strategy in order to provide spatial bounds for the switched system.

Systems with bifurcation often contain multiple $\omega$-limit sets which cannot be globally exponentially stable. Instead, results such as LaSalle's invariant set theorem [10] allow us to analyze asymptotic stability of this larger class of systems. LaSalle's theorem and much of the switched systems literature are both Lyapunov-based, and we will make use of Lyapunov functions to define all the relevant sets.

Section 2 provides background assumptions and definitions. Section 3 begins by reconsidering existing results. Sections 3.3 through 3.5 present two methods to accomplish the goal. Choice of a particular method will depend on specific situations and design constraints. Section 4 shows two numerical examples, and Section 5 provides concluding comments.

\section{Preliminaries and Definitions}

Consider a set of continuous-time dynamical systems defined by

$$
\dot{\mathbf{x}}=\mathbf{f}_{p}(\mathbf{x}),
$$

where $\mathbf{x} \in \mathbb{R}^{n}$ and $p \in \mathcal{P}$, with some index set $\mathcal{P}=\left\{p_{1}, p_{2}, \ldots, p_{\max }\right\}$. A piecewise constant switching signal $\sigma:[0, \infty) \rightarrow \mathcal{P}$ specifies the active subsystem at each time. Assume, for ease of analysis, that $\mathbf{f}_{p}$ are each continuous with continuous first partials. Together, (2), the index set, and the switching signal define a switched system.

We will consider a constraint on how quickly the switching signal can make consecutive switches. 
Definition Consider a switched system with switching times $\left\{t_{1}, t_{2}, \ldots\right\}$. It is said to have dwell time $\tau$ if $t_{i+1}-t_{i} \geq \tau \forall i \in \mathbb{N}$.

In this paper, integer subscripts on $t$ are reserved for switching times. Denote the limit from the right/left as superscript $+/-$, respectively.

Next, we review and introduce some important subsets of $\mathbb{R}^{n}$. Assume that each subsystem has a (possibly different) $\mathcal{C}^{1}$ Lyapunov-like function, which is bounded above and below on every bounded subset of $\mathbb{R}^{n}$. Furthermore, assume that each is radially unbounded $\left(V_{p}(\mathbf{x}) \rightarrow \infty\right.$ as $\left.\|\mathbf{x}\| \rightarrow \infty\right)$. This ensures that every sublevel set describes a compact region. We assume for the remainder of this paper that the minimum value of each $V_{p}$ is zero. Define

$$
\mathcal{G}_{p}=\left\{\mathbf{x} \in \mathbb{R}^{n} \mid V_{p}(\mathbf{x})=0\right\}
$$

as the set which attains the minimum value of $V_{p}$. Let $\kappa$ be a positive constant and define

$$
\mathcal{N}_{p}(\kappa)=\left\{\mathbf{x} \in \mathbb{R}^{n} \mid V_{p}(\mathbf{x}) \leq \kappa\right\}
$$

a closed $\kappa$-neighborhood of $\mathcal{G}_{p}$. For the purposes of Theorem $1, \mathcal{N}_{p}(\kappa)$ is connected, but it is not necessarily connected in the remainder of the paper (See Figure 4). Let

$$
\mathcal{N}(\kappa)=\bigcup_{p \in \mathcal{P}} \mathcal{N}_{p}(\kappa) .
$$

Additionally, define a superset, $\mathcal{M}(\kappa)$, in a series of steps with

$$
\alpha_{p}(\kappa)=\max _{\mathbf{x} \in \mathcal{N}(\kappa)} V_{p}(\mathbf{x})
$$

and

$$
\mathcal{M}_{p}(\kappa)=\left\{\mathbf{x} \in \mathbb{R}^{n}: V_{p}(\mathbf{x}) \leq \alpha_{p}(\kappa)\right\} .
$$

Finally, we create a closed union of closed sublevel sets,

$$
\mathcal{M}(\kappa)=\bigcup_{p \in \mathcal{P}} \mathcal{M}_{p}
$$

Notice that the dependence on $\kappa$ carries through once we use it in $\mathcal{N}(\kappa)$. For the purposes of Theorem $1, \mathcal{M}$ is a connected superset of $\mathcal{N}$. Theorem 4 will introduce a different notion which is not necessarily connected. Figure 2 provides a one-dimensional example to help visualize these sets. 


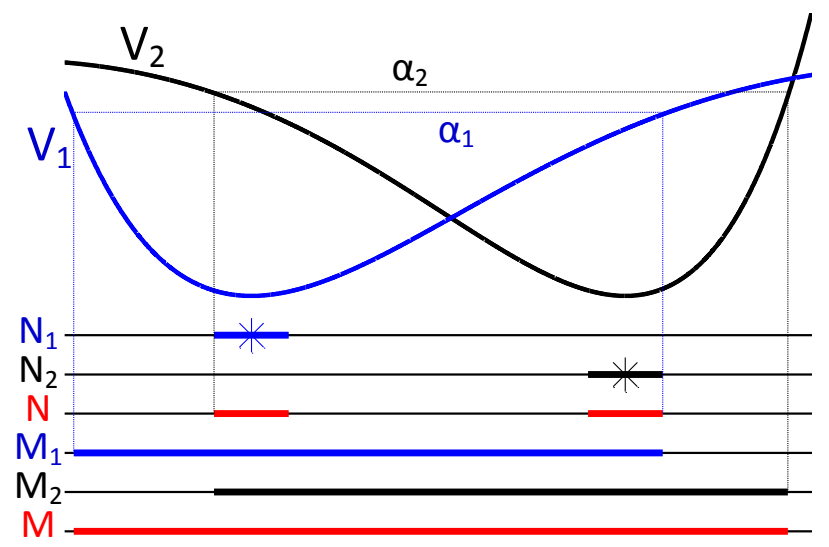

Figure 2: Qualitative example of how $\mathcal{N}$ and $\mathcal{M}$ are built for a switched system consisting of two subsystems, each with a single equilibrium, but at different locations.

\section{Stability Results}

We first restructure the result in [9] to add clarity and to better facilitate the generalization presented in this paper.

\subsection{Unique Equilibrium Case}

Theorem 1. Consider a family of systems defined by (2), each with a single, globally exponentially stable equilibrium. Suppose that the exponential decay rate of each Lyapunov function, as described in

$$
\dot{V}_{p}(\mathbf{x}) \leq-\varepsilon V_{p}(\mathbf{x}), \forall \mathbf{x} \in \mathbb{R}^{n}, \forall p \in \mathcal{P},
$$

is at least $\varepsilon>0$.

Furthermore, given a positive constant $\kappa$, define the sets as in Section 2 and assume $\mu(\kappa) \in(1, \infty)$ such that

$$
\frac{V_{r}(\mathbf{x})}{V_{q}(\mathbf{x})} \leq \mu(\kappa), \forall q, r \in \mathcal{P} \quad \forall \mathbf{x} \in \mathbb{R}^{n} \backslash \mathcal{N}(\kappa) .
$$

Then, for every switching signal with dwell time

$$
\tau>\frac{\log \mu(\kappa)}{\varepsilon},
$$

the following three statements hold: 
(i) For any switching time $t_{i}$ such that $\mathbf{x}\left(t_{i}\right) \in \mathcal{N}$, there exists a time $\tilde{t} \in\left[t_{i}, t_{i+1}\right)$ such that $\mathbf{x}(\tilde{t}) \in \mathcal{N}_{\sigma(\tilde{t})}$.

(ii) For any initial condition, there exists a time $T$ such that $\mathbf{x}\left(T^{-}\right) \in$ $\mathcal{N}_{\sigma\left(T^{-}\right)}(\kappa)$, and

(iii) For any time $\bar{t}$ such that $\mathbf{x}(\bar{t}) \in \mathcal{N}_{\sigma(\bar{t})}(\kappa), \mathbf{x}(t) \in \mathcal{M}(\kappa)$ for all $t \geq \bar{t}$.

Proof. The proof proceeds in three parts:

(i) Given $\mathbf{x}\left(t_{i}\right) \in \mathcal{N}$, consider the connected component $\mathcal{Y} \subset \mathcal{N}$ that contains $\mathbf{x}\left(t_{i}\right)$. There are two potential subcases.

(1) It may be possible that $V_{\sigma\left(t_{i}^{+}\right)}\left(\mathbf{x}\left(t_{i}^{+}\right)\right)>V_{\sigma\left(t_{i}^{+}\right)}(\mathbf{y})$ for all $\mathbf{y} \in \partial \mathcal{Y}$ (the boundary of $\mathcal{Y})$. Since $V_{\sigma\left(t_{i}^{+}\right)}$is continuous, this implies that $V_{\sigma\left(t_{i}^{+}\right)}$has a local maximum in the interior of $\mathcal{Y}$ with $\nabla V_{\sigma\left(t_{i}^{+}\right)}=0$ and $\dot{V}_{\sigma\left(t_{i}^{+}\right)}=0$ at that point. We have reached a contradiction.

(2) The remaining case is that there exists a $\mathbf{y} \in \partial \mathcal{Y}$ such that for some $r$

$$
V_{\sigma\left(t_{i}^{+}\right)}\left(\mathbf{x}\left(t_{i}^{+}\right)\right) \leq V_{\sigma\left(t_{i}^{+}\right)}(\mathbf{y}) \leq \mu V_{r}(\mathbf{y})=\mu \kappa .
$$

From the appendix, Proposition 7(1) along with continuity of $V_{p}$ verifies the second inequality, while Proposition 7(2) and Proposition 8 verify the final equality. Then,

$$
V_{\sigma\left(t_{i+1}^{-}\right)}\left(\mathbf{x}\left(t_{i+1}\right)\right) \leq e^{-\varepsilon\left(t_{i+1}-t_{i}\right)} \mu \kappa,
$$

and the dwell time given in (11) is sufficient to force the trajectory into $\mathcal{N}_{\sigma\left(t_{i}^{+}\right)}$before time $t_{i+1}$.

(ii) Consider a finite time interval $\left[t_{0}, T\right]$ with corresponding switching times $t_{1}<t_{2}<\cdots<t_{n_{\sigma}}$, where $n_{\sigma}$ is the number of switches inside the interval. Between switches, $\sigma(t)$ is constant. If the trajectory enters $\mathcal{N}_{\sigma(t)}$ (not just $\left.\mathcal{N}\right)$, the result is trivial. Otherwise, the behavior of $V_{\sigma(t)}(\mathbf{x}(t))$ between switches satisfies (9). That is,

$$
V_{\sigma\left(t_{i+1}^{-}\right)}\left(\mathbf{x}\left(t_{i+1}^{-}\right)\right) \leq e^{-\varepsilon\left(t_{i+1}-t_{i}\right)} V_{\sigma\left(t_{i}^{+}\right)}\left(\mathbf{x}\left(t_{i}^{+}\right)\right) .
$$

At switches, there are two cases.

(1) For some $i, \mathbf{x}\left(t_{i}\right) \in \mathcal{N}$. Part (i) applies, and the trajectory will enter $\mathcal{N}_{\sigma(t)}$ before $t_{i+1}$. 
(2) For every $i, \mathbf{x}\left(t_{i}\right) \in \mathbb{R}^{n} \backslash \mathcal{N}$. Now,

$$
V_{\sigma\left(t_{i}^{+}\right)}\left(\mathbf{x}\left(t_{i}^{+}\right)\right) \leq \mu(\kappa) V_{\sigma\left(t_{i}^{-}\right)}\left(\mathbf{x}\left(t_{i}^{-}\right)\right)
$$

holds. We can iterate on $i$ to obtain

$$
V_{\sigma\left(T^{-}\right)}\left(\mathbf{x}\left(T^{-}\right) \leq e^{((\log \mu(\kappa) / \tau)-\varepsilon)\left(T-t_{0}\right)} V_{\sigma\left(t_{0}\right)}\left(\mathbf{x}\left(t_{0}\right)\right) .\right.
$$

Under the dwell time condition, (11), this implies that by taking $T$ suitably large, we can make $V_{\sigma\left(T^{-}\right)}\left(\mathbf{x}\left(T^{-}\right)\right)$arbitrarily small. Thus, $\mathbf{x}\left(T^{-}\right) \in \mathcal{N}_{\sigma\left(T^{-}\right)}(\kappa)$. This proof is existential, not constructive. We cannot calculate a particular time $T$ for any particular problem.

(iii) Since $\mathcal{N}_{\sigma(\bar{t})}$ is a Lyapunov sub-level set, the trajectory cannot exit until a switch occurs; call the time of this switch $t_{i}$. Part (i) gives that the trajectory enters $\mathcal{N}_{\sigma\left(t_{i}^{+}\right)}$before $t_{i+1}$. Furthermore, $\mathbf{x}\left(t_{i}\right) \in \mathcal{N}_{\sigma\left(t_{i}^{-}\right)} \subset$ $\mathcal{M}_{\sigma\left(t_{i}^{+}\right)}$, which is forward invariant while the $\sigma\left(t_{i}^{+}\right)$system is active.

The proof presented above is different from [9] in two important ways. First, we explicitly handle the case when a switch occurs inside $\mathcal{N} \backslash \mathcal{N}_{\sigma(t)}$ (where (10) does not hold). Second, we require that the trajectory enters $\mathcal{N}_{\sigma(t)}$ rather than $\mathcal{N}$ before we can conclude that it never exits $\mathcal{M}$. In fact, it is an error to do otherwise. The trajectory may pass through $\mathcal{N} \backslash \mathcal{N}_{\sigma(t)}$ and then switch after it has emerged, which may cause it to exit $\mathcal{M}$. We will demonstrate with an example.

Example This example is a slight variation of Example 2 from [9]. Choose

$$
\dot{\mathbf{x}}_{p}=A \mathbf{x}_{p}+b_{p}
$$

but with

$$
\begin{gathered}
A=\left[\begin{array}{cc}
-1 & -10 \\
10 & -1
\end{array}\right] \\
b_{1}=\left[\begin{array}{c}
10 \\
1
\end{array}\right], b_{2}=\left[\begin{array}{c}
-1 \\
10
\end{array}\right], b_{3}=\left[\begin{array}{c}
1 \\
-10
\end{array}\right] .
\end{gathered}
$$

We are able to use the same Lyapunov functions as [9], $V_{1}(\mathbf{x}):=x_{1}^{2}+\left(x_{2}-\right.$ $1)^{2}, V_{2}(\mathbf{x}):=\left(x_{1}+1\right)^{2}+x_{2}^{2}$, and $V_{3}(\mathbf{x}):=\left(x_{1}-1\right)^{2}+x_{2}^{2}$. One can check that $\tau$ 


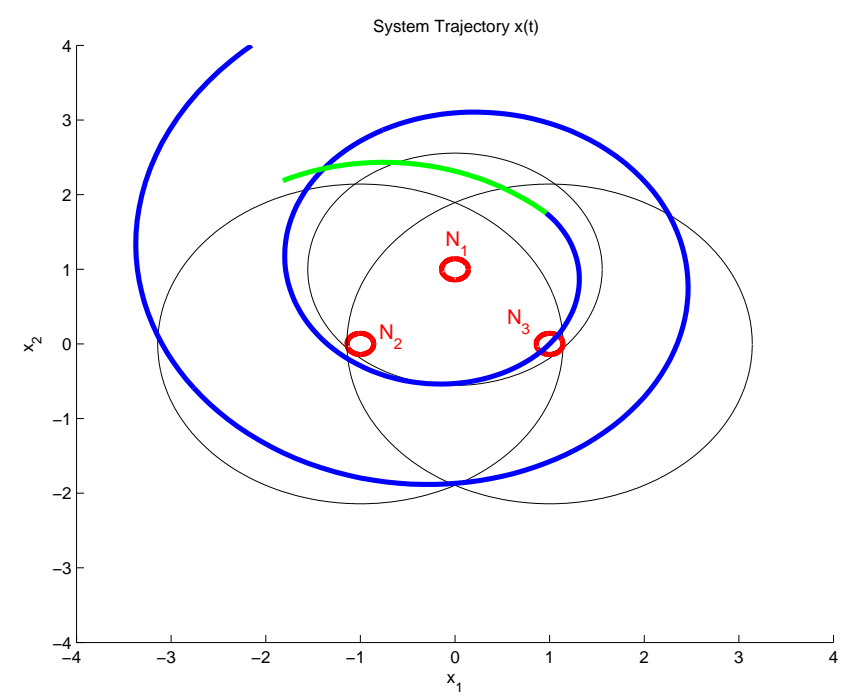

Figure 3: Example trajectory demonstrating the need for time-dependent $\mathcal{N}_{\sigma(t)}(\kappa)$.

can be the same as in [9]. Consider the trajectory shown in Figure 3. The red circles show the boundaries for $\mathcal{N}_{p}$; the black circles show the boundaries for $\mathcal{M}_{p}$. Subsystem 1 is active at the start, and the trajectory is shown in blue. $\mathcal{N}_{1}$ is the red circle centered around $\left[\begin{array}{ll}0 & 1\end{array}\right]^{T}$. The trajectory passes through $\mathcal{N}_{3}$, which is the red circle centered around $\left[\begin{array}{ll}1 & 0\end{array}\right]^{T}$. This is entering $\mathcal{N}$, but not $\mathcal{N}_{\sigma(t)}$. Now, notice that we could have selected initial conditions to make the entry into $\mathcal{N}_{3}$ occur at arbitrarily large time. This allows us to place a switch anywhere along the trajectory, regardless of $\tau$ (in particular, we will place it outside of $\mathcal{N}$ ). In this case, we switched to subsystem 2 at the location where the trajectory changes to green. It exits $\mathcal{M}$ shortly thereafter.

Remark 1. Not all choices of $V_{p}$ will give a finite value of $\mu(\kappa)$. Typical polynomial constructions for Lyapunov functions must have the same polynomial order. Scaling or stretching Lyapunov functions may be useful, but there will be implications for both the spatial parameter $\mu$ and the temporal parameter $\varepsilon$. We will will revisit the idea of scaling briefly in Sections 3.3-3.4.

\subsection{Problem Statement: Switching Systems having Multiple Invariant Sets}

Alpcan and Başar considered subsystems, each having a globally exponentially stable equilibrium point [9]. We will relax this condition to allow 
for systems with multiple invariant sets. Consider a switched system with $C^{1}$ functions $V_{p}: \mathbb{R}^{n} \rightarrow \mathbb{R}$ bounded on every bounded subset of $\mathbb{R}^{n}$ such that

$$
\dot{V}_{p}(\mathbf{x}) \leq 0, \forall \mathbf{x} \in \mathbb{R}^{n},
$$

and $V_{p}(\mathbf{x}) \rightarrow \infty$ as $\|\mathbf{x}\| \rightarrow \infty$. Denote

$$
E_{p}=\left\{\mathbf{x} \in \mathbb{R}^{n} \mid \dot{V}(\mathbf{x})=0\right\} .
$$

Our problem is as follows. Is there a dwell time condition that suffices for ultimate boundedness?

Two challenges are immediately apparent. First, if $\dot{V}_{p}$ vanishes outside of $\mathcal{N}_{p}$, there is not a strictly positive Lyapunov decay rate outside of $\mathcal{N}_{p}$, so it is unclear how long to wait between switches. Second, even in the absence of switching, the active system may never enter $\mathcal{N}_{p}\left(E_{p}\right.$ may not be contained in $\mathcal{N}_{p}$; see Figure 4). The following three subsections describe two methods for overcoming these challenges.

\subsection{Intermediate Solution: Expand Entry Neighborhood}

The simplest idea is to realize that $\mathcal{N}_{p}(\kappa)$ grows in size as $\kappa$ increases. Assuming that $E_{p}$ is bounded, we can pick $\kappa$ large enough so that $E_{p} \subset \mathcal{N}_{p}(\kappa)$. Even so, not all Lyapunov functions satisfying (19) will decay exponentially on $\mathbb{R}^{n} \backslash \mathcal{N}_{p}(\kappa)$. For example, consider a single one-dimensional subsystem $\dot{x}=-\arctan (x)$ with $V=x^{2}$. Nevertheless, the following lemma is useful:

Lemma 2. Consider a switched system with Lyapunov functions $\mathcal{V}_{p}$ satisfying $\dot{\mathcal{V}}_{p} \leq 0$ with $\mathcal{V}_{p}(\mathbf{x}) \rightarrow \infty$ as $\|\mathbf{x}\| \rightarrow \infty$ and $E_{p}$ bounded. Then, for sublevel Lyapunov sets $\mathcal{N}_{p}(\kappa)$ such that $E_{p} \subset \operatorname{Int}\left(\mathcal{N}_{p}(\kappa)\right.$ ) (where Int denotes the interior) and any $\varepsilon \in \mathcal{R}$, there exists a different set of continuous, radially unbounded Lyapunov functions $V_{p}$ such that,

1. $V_{p}(\mathbf{x})=0$ for $\mathbf{x} \in \mathcal{N}_{p}$, and $V_{p}(\mathbf{x})>0$ for $\mathbf{x} \in \mathbb{R}^{n} \backslash \mathcal{N}_{p}$.

2. $V_{p}\left(\mathbf{x}\left(t+t_{0}\right)\right) \leq e^{-\varepsilon t} V_{p}\left(\mathbf{x}\left(t_{0}\right)\right)$ for all $\mathbf{x} \in \mathbb{R}^{n} \backslash \mathcal{N}_{p}$ and $t, t_{0} \in \mathbb{R}$.

Proof. Bhatia [11] constructed a unique continuous function $s(\mathbf{x})$ on $\mathbb{R}^{n} \backslash \mathcal{N}_{p}$ such that $s\left(\mathbf{x}\left(t+t_{0}\right)\right)=s\left(\mathbf{x}\left(t_{0}\right)\right)-t$ and $s(\mathbf{x}) \rightarrow 0$ as $\mathbf{x} \rightarrow \mathcal{N}_{p}$. Then, we can select any constant $\varepsilon$ and set

$$
V_{p}(\mathbf{x})= \begin{cases}0 & \text { for } \mathbf{x} \in \mathcal{N}_{p} \\ e^{\varepsilon s(\mathbf{x})} & \text { for } \mathbf{x} \in \mathbb{R}^{n} \backslash \mathcal{N}_{p}\end{cases}
$$


Hence, on $\mathbb{R}^{n} \backslash \mathcal{N}_{p}$,

$$
V_{p}\left(\mathbf{x}\left(t+t_{0}\right)\right)=e^{\varepsilon s\left(\mathbf{x}\left(t+t_{0}\right)\right)}=e^{\varepsilon\left(s\left(\mathbf{x}\left(t_{0}\right)\right)-t\right)}=e^{-\varepsilon t} V_{p}\left(\mathbf{x}\left(t_{0}\right)\right) .
$$

This means that for suitably large $\kappa$, there exists $V_{p}$ that decays exponentially outside $\mathcal{N}_{p}(\kappa)$. A simple constant shift can patch the original Lyapunov function on $\mathcal{N}_{p}(\kappa)$ with the construction of Lemma 2 outside $\mathcal{N}_{p}(\kappa)$ at $\partial \mathcal{N}_{p}$ (the boundary of $\mathcal{N}_{p}$ ), and the mere continuity of $V_{p}$ outside of $\mathcal{N}_{p}$ does not harm any essential parts of the proof. (Note that performing a constant shift outside $\mathcal{N}_{p}(\kappa)$ will scale $\varepsilon$ by $\alpha_{p}$, but we could just perform the construction again with a larger $\varepsilon$ to correct for this). However, relying on the construction of Lemma 2 may not allow for (10) to hold, and we must assume that we can find a set of Lyapunov functions which satisfy both the exponential decay property and the $\mu$ property, (10). It is left as an open problem to determine if it is generally possible to construct a set of Lyapunov functions that satisfy both conditions for any switched system.

Now, the conditions required for Theorem 1 hold (noting that is is not essential to have exponential Lyapunov decay within $\mathcal{N}_{p}$ ).

Remark 2. The set definitions do not require a single value of $\kappa$ for all subsystems. If our subsystems have qualitatively different invariant sets, a single $\kappa$ may produce large sets or dwell time. Instead, we can choose a set, $\boldsymbol{\kappa}=\left\{\kappa_{p}\right\}$, of values and restate all our set definitions as functions of $\boldsymbol{\kappa}$.

Making $\kappa_{p}$ large is problematic in two ways. First, our analysis says little about the trajectory inside $\mathcal{N}(\boldsymbol{\kappa})$. However, with large $\kappa_{p}$, this region may be large. Second, it may lead to larger dwell time or larger $\mathcal{M}(\boldsymbol{\kappa})$. In the next two subsections, we describe a method to more tightly tailor our strategy.

\subsection{First Main Result: Tightly Tailored Entry Set}

Since the main regions of concern are those where $\dot{V}_{p}$ is closest to zero, we will now define a smaller set containing these regions. Choose a set $\boldsymbol{\delta}=\left\{\delta_{p_{1}}, \delta_{p_{2}}, \ldots, \delta_{p_{\max }}\right\}$ with $\delta_{p_{i}}>0$, and define the set

$$
\mathcal{H}_{p}\left(\delta_{p}\right)=\left\{\mathbf{x} \in \mathbb{R}^{n}: \dot{V}_{p}(\mathbf{x})>-\delta_{p}\right\}
$$


so that $E_{p} \subset \mathcal{H}_{p}$. As usual, we can also define $\mathcal{H}(\boldsymbol{\delta})=\bigcup_{p \in \mathcal{P}} \mathcal{H}_{p}$. Figure 4 provides a one-dimensional example to help visualize how these sets are constructed. Similar to before, not all $V_{p}$ satisfying (19) decay exponentially outside $\mathcal{H}_{p}\left(\delta_{p}\right)$. Define

$$
\gamma_{p}\left(\delta_{p}\right)=\max _{\mathbf{x} \in \mathcal{H}_{p}} V_{p}(\mathbf{x})
$$

with

$$
\gamma(\boldsymbol{\delta})=\max _{p \in \mathcal{P}} \gamma_{p}\left(\delta_{p}\right)
$$

Furthermore, set

$$
\mathcal{L}_{p}\left(\delta_{p}\right)=\left\{\mathbf{x} \in \mathbb{R}^{n}: V_{p}(\mathbf{x}) \leq \gamma_{p}\left(\delta_{p}\right)\right\} .
$$

Since $\mathcal{L}_{p}$ is a sublevel Lyapunov set, it is compact. From compactness, $\dot{V}_{p}$ attains a minimum on $\mathcal{L}_{p}$, while $V_{p}$ attains a finite maximum. Thus, on $\mathcal{L}_{p} \backslash$ $\mathcal{H}_{p}$, an exponential decay rate can be computed, while we can use Lemma 2 outside $\mathcal{L}_{p}$. Again, if necessary, a simple constant shift can patch the two functions together at $\partial \mathcal{L}_{p}$.

While the construction in Lemma 2 only gives $s(\mathbf{x})$ continuous in the multi-dimensional sense, it is clearly directionally differentiable along the subsystem trajectories. Thus, writing $\dot{V}$ is sound notation. Putting it all together, we can compute $\varepsilon$ such that

$$
\dot{V}_{p}(\mathbf{x}) \leq-\varepsilon V_{p}(\mathbf{x}), \forall \mathbf{x} \in \mathbb{R}^{n} \backslash \mathcal{H}_{p}\left(\delta_{p}\right), \forall p \in \mathcal{P} .
$$

Unfortunately, $\mathcal{H}_{p}$ may be disconnected, and it is not necessarily invariant even in the absence of switching. We will engage these problems directly in Sec. 3.5, but for now, define

$$
\eta_{p}(\boldsymbol{\delta})=\sup _{\mathbf{x} \in \mathcal{H}} V_{p}(\mathbf{x}),
$$

with the usual $\eta(\boldsymbol{\delta})=\max _{p \in \mathcal{P}} \eta_{p}\left(\delta_{p}\right)$, and we can proceed directly to a theorem demonstrating the usefulness of embedding $\mathcal{N}$ inside $\mathcal{H}$.

Theorem 3. Consider a family of systems defined by (2), each with a radially unbounded Lyapunov-like function that satisfies (19). Assume $\mathcal{H}_{p}, E_{p}$ bounded and $\mathcal{G}_{p} \subset \mathcal{H}_{p}\left(\delta_{p}\right)$. Compute $\varepsilon(\boldsymbol{\delta})$ so that (27) is satisfied and assume 


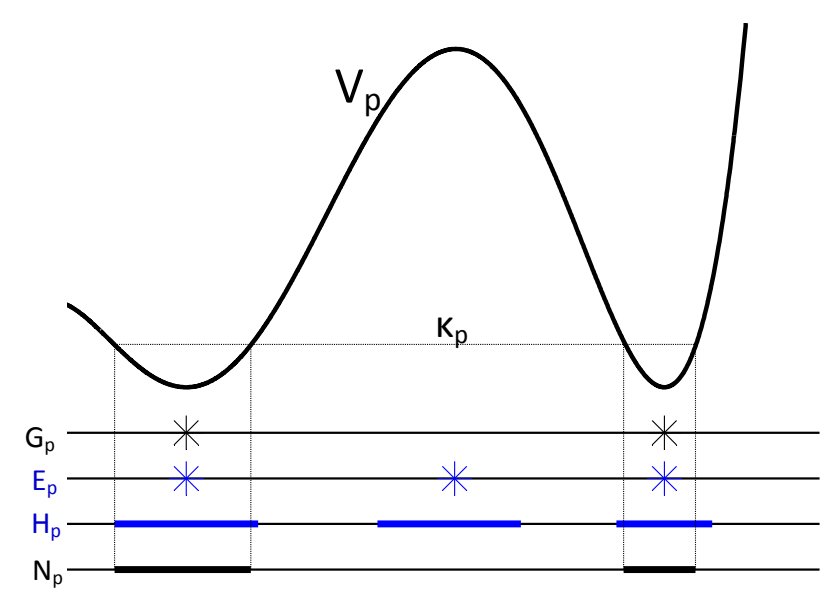

Figure 4: Qualitative example of tighter tailoring. The subsystem has two stable equilibria and a single unstable equilibrium. Notice that $\mathcal{N}_{p} \subset \mathcal{H}_{p}$.

$$
\frac{V_{r}(\mathbf{x})}{V_{q}(\mathbf{x})} \leq \mu(\boldsymbol{\delta}), \forall q, r \in \mathcal{P}, \quad \forall \mathbf{x} \in \mathbb{R}^{n} \backslash \mathcal{H}(\boldsymbol{\delta})
$$

holds for finite $\mu(\boldsymbol{\delta})$. Furthermore, compute $\kappa_{p}>0$ such that $\mathcal{G}_{p} \subset \mathcal{N}_{p}\left(\kappa_{p}\right) \subseteq$ $\mathcal{H}_{p}\left(\delta_{p}\right)$. Set $\kappa=\min _{p \in \mathcal{P}} \kappa_{p}$. Then, for every switching signal with dwell time

$$
\tau>\frac{\log \max \left(\mu, \frac{\eta}{\kappa}\right)}{\varepsilon},
$$

there exists a time $T$ such that $\mathbf{x}\left(T^{-}\right) \in \mathcal{H}_{\sigma\left(T^{-}\right)}(\boldsymbol{\delta})$.

Proof. If the trajectory enters $\mathcal{H}_{\sigma(t)}$ (not just $\mathcal{H}$ ), the result is trivial. Otherwise, the behavior of $V_{\sigma(t)}(\mathbf{x}(t))$ between switches satisfies (27). That is,

$$
V_{\sigma\left(t_{i+1}^{-}\right)}\left(\mathbf{x}\left(t_{i+1}^{-}\right)\right) \leq e^{-\varepsilon\left(t_{i+1}-t_{i}\right)} V_{\sigma\left(t_{i}^{+}\right)}\left(\mathbf{x}\left(t_{i}^{+}\right)\right) .
$$

At switches, there are two cases.

(1) For some $i, \mathbf{x}\left(t_{i}\right) \in \mathcal{H}$. Then,

$$
V_{\sigma\left(t_{i+1}^{-}\right)}\left(\mathbf{x}\left(t_{i+1}\right)\right) \leq e^{-\varepsilon\left(t_{i+1}-t_{i}\right)} V_{\sigma\left(t_{i}^{+}\right)}\left(\mathbf{x}\left(t_{i}^{+}\right)\right) \leq e^{-\varepsilon\left(t_{i+1}-t_{i}\right)} \eta,
$$

and the dwell time given in (30) is sufficient to force the trajectory into $\mathcal{N}_{\sigma\left(t_{i}^{+}\right)} \subset \mathcal{H}_{\sigma\left(t_{i}^{+}\right)}$before time $t_{i+1}$. The trajectory may enter $\mathcal{H}_{\sigma\left(t_{i}^{+}\right)}$and not $\mathcal{N}_{\sigma\left(t_{i}^{+}\right)}$, but that is sufficient. 
(2) For every $i, \mathbf{x}\left(t_{i}\right) \in \mathbb{R}^{n} \backslash \mathcal{H}$. Then, (29) holds, and we can iterate just as in (16) to achieve the same result.

Remark 3. In [9], $\kappa$ was a single tuning parameter. In Section 3.3, $\boldsymbol{\kappa}=\left\{\kappa_{p}\right\}$ was introduced as a possible set of tuning parameters. Now, per Theorem 3 , the set of tuning parameters is $\boldsymbol{\delta}=\left\{\delta_{p}\right\}$, and $\boldsymbol{\kappa}$ is computed as a consequence of our selection of $\boldsymbol{\delta}$. Since nearly every parameter/set which follows is dependent on $\boldsymbol{\delta}$, we will often omit explicit dependence in favor of readability.

\subsection{Second Main Result: No-Escape Set}

This section assumes the trajectory has entered $\mathcal{H}_{\sigma(t)}(\boldsymbol{\delta})$ at some time and proceeds to build the relevant no-escape set, which will be denoted $\mathcal{M}_{p}$. The primary problem is that $\mathcal{H}_{p}$ is not necessarily an invariant set even for periods of time when $\sigma(t)$ is constant (i.e., no switching).

Example Consider two one-dimensional subsystems, $\dot{x}_{1}=-x_{1}$ and $\dot{x}_{2}=$ $x_{2}-x_{2}^{3}$, with $V_{2}=(x-1)^{2}(x+1)^{2}$ and small $\delta_{2}$. Assume a nonzero initial condition with the first subsystem being active. The trajectory can become arbitrarily close to zero before switching to the second subsystem. While the second subsystem is active $(\sigma(t)$ is constant), the trajectory can clearly leave $\mathcal{H}_{2}$. Furthermore, since the second subsystem started arbitrarily close to the origin, it can take an arbitrarily long time to exit $\mathcal{H}_{2}$. Thus, no finite dwell time can prevent at least one switch from being possible outside of $\mathcal{H}$.

Theorem 4. Consider a family of systems defined by (2), each with a radially unbounded Lyapunov-like function that satisfies (19). Assume $\mathcal{H}_{p}, E_{p}$ bounded and $\mathcal{G}_{p} \subset \mathcal{H}_{p}(\boldsymbol{\delta})$. Compute $\varepsilon(\boldsymbol{\delta})$ so that (27) and (29) hold. Furthermore, compute $\kappa_{p}>0$ such that $\mathcal{G}_{p} \subset \mathcal{N}_{p}(\boldsymbol{\kappa}) \subseteq \mathcal{H}_{p}(\boldsymbol{\delta})$. Set $\mathcal{L}(\boldsymbol{\delta})=\bigcup_{p \in \mathcal{P}} \mathcal{L}_{p}$. Compute

$$
\xi_{p}(\boldsymbol{\delta})=\max _{\mathbf{x} \in \mathcal{L}(\boldsymbol{\delta})} V_{p}(\mathbf{x}),
$$

and set $\xi(\boldsymbol{\delta})=\max _{p \in \mathcal{P}} \xi_{p}(\boldsymbol{\delta})$. Then, for every switching signal with dwell time

$$
\tau>\frac{\log \frac{\xi}{\kappa}}{\varepsilon},
$$


for every $\bar{t}$ such that $\mathbf{x} \in \mathcal{H}_{\sigma(\bar{t})}, \mathbf{x}(t) \in \mathcal{M}(\boldsymbol{\delta})=\bigcup_{p \in \mathcal{P}} \mathcal{M}_{p}$ for all $t \geq \bar{t}$, where

$$
\mathcal{M}_{p}(\boldsymbol{\delta})=\left\{\mathbf{x} \in \mathbb{R}^{n}: V_{p}(\mathbf{x}) \leq \xi_{p}\right\} .
$$

Proof. Consider the following sequence of times. Assume a switch occurs or the system is started at $t_{0}$. Assume further that there is a time $t_{0} \leq t_{\text {enter }}$ at which the trajectory enters $\mathcal{H}_{\sigma\left(t_{0}^{+}\right)}(\boldsymbol{\delta})$. There may or may not be a time at which the trajectory exits $\mathcal{H}_{\sigma\left(t_{0}^{+}\right)}(\boldsymbol{\delta})$, which we will denote $t_{\text {enter }} \leq t_{\text {exit }}$. If there is, $t_{\text {exit }}-t_{\text {enter }}$ could possibly be arbitrarily large. Therefore, the second switching time $t_{1}$ may be shortly after $t_{\text {exit }}$ while the trajectory is outside of $\mathcal{H}_{\sigma\left(t_{0}^{+}\right)}(\boldsymbol{\delta})$ regardless of the dwell time.

The proof will proceed in two parts. First, we will show that the trajectory does not leave $\mathcal{M}$ before a third switching time, called $t_{2}$. Then, we will compute a dwell time so that the trajectory must re-enter $\mathcal{H}_{\sigma(t)}$ before $t_{2}$. By induction, the trajectory will never leave $\mathcal{M}$.

(i) We will show that the trajectory never leaves $\mathcal{M}$ on $\left[t_{\text {enter }}, t_{1}\right]$, then on $\left[t_{1}, t_{2}\right]$. Since $\mathbf{x}\left(t_{\text {enter }}\right) \in \mathcal{H}_{\sigma\left(t_{0}\right)} \subset \mathcal{M}_{\sigma\left(t_{0}\right)}$ and $\mathcal{M}_{\sigma\left(t_{0}\right)}$ is a sublevel Lyapunov set, the trajectory cannot leave $\mathcal{M}_{\sigma\left(t_{0}\right)}$ on $\left[t_{\text {enter }}, t_{1}\right]$ since no switching occurs on this interval. Next, notice

$$
\mathbf{x}\left(t_{1}\right) \in \mathcal{L}_{\sigma\left(t_{1}^{-}\right)} \subset \mathcal{L} \subset \mathcal{M}_{\sigma\left(t_{1}^{+}\right)}
$$

Thus, the trajectory cannot leave $\mathcal{M}_{\sigma\left(t_{1}^{+}\right)}$on $\left[t_{1}, t_{2}\right]$.

(ii) We will show that the trajectory must re-enter $\mathcal{H}_{\sigma(t)}$ before $t_{2}$. Define $\Delta=t_{2}-t_{1} \geq \tau$, giving

$$
V_{\sigma\left(t_{2}^{-}\right)}\left(\mathbf{x}\left(t_{2}^{-}\right)\right) \leq e^{-\varepsilon \Delta} V_{\sigma\left(t_{1}^{+}\right)}\left(\mathbf{x}\left(t_{1}^{+}\right)\right) \leq e^{-\varepsilon \Delta} \xi_{\sigma\left(t_{1}^{+}\right)} \leq e^{-\varepsilon \Delta} \xi .
$$

The dwell time given by (34) ensures $V_{\sigma\left(t_{2}^{-}\right)}(\mathbf{x})\left(t_{2}^{-}\right) \leq \kappa$. Thus, the trajectory enters $\mathcal{N}_{\sigma\left(t_{1}^{+}\right)}$or reenters $\mathcal{H}_{\sigma(t)}$ elsewhere before time $t_{2}$.

Section 4 will show examples that have $\frac{\xi}{\kappa}>\mu$, but it is trivial to construct cases with very large $\mu$. Next, we consider whether the bounds in these theorems are tighter than some other possible bounds.

Proposition 5. Given the preceding set definitions, $\mu \gamma \geq \xi_{p} \geq \eta_{p} \forall p$. 
Proof. Since $\mathcal{H}_{p} \subset \mathcal{L}_{p}, \mathcal{H} \subset \mathcal{L}$, and $\xi_{p} \geq \eta_{p}$. Without loss of generality, consider $\mathbf{x} \in \mathcal{L}$ such that $V_{1}(\mathbf{x})=\xi_{1}$. There are three cases:

Case 1. Suppose $\mathbf{x} \in \mathcal{H}_{1}$. Then, $\xi_{1}=\gamma_{1}$. Since $\gamma \geq \gamma_{1}$ and $\mu \geq 1, \mu \gamma \geq \xi_{1}$.

Case 2. Suppose $\mathbf{x} \in \mathcal{H} \backslash \mathcal{H}_{1}$. Then, $\xi_{1}=\eta_{1}$. Since $\xi_{1}$ is a maximum of $V_{1}$ over $\mathcal{L} \supset \mathcal{H}$ and $H$ is open, $V_{1}(\mathbf{x})$ must be a local maximum. But, if it is a local maximum, $\nabla V_{1}(\mathbf{x})=0$ and $\dot{V}_{1}(\mathbf{x})=0$. Thus, $\mathbf{x} \in \mathcal{H}_{1}$, and the problem is reduced to Case 1.

Case 3. Suppose $\mathbf{x} \in \mathcal{L} \backslash \mathcal{H}$. Then, (29) holds, and

$$
\mu \geq \frac{V_{1}(\mathbf{x})}{V_{p}(\mathbf{x})}, \quad \forall p .
$$

Then, for some $p, \mu \gamma \geq \mu \gamma_{p} \geq \mu V_{p}(\mathbf{x}) \geq V_{1}(\mathbf{x})=\eta_{1}=\xi_{1}$.

Corollary 6. Taken together, Theorems 3 and 4 provide a dwell time which guarantees finite time entry into $\mathcal{H}_{\sigma(t)}$ and the invariance of $\mathcal{M}$ thereafter. Given a particular problem, if we want to apply both theorems, we should set $\tau>\frac{\log \max \left(\mu, \frac{\xi}{\kappa}\right)}{\varepsilon}$.

Notice that in the ideal case, with each subsystem consisting only of a single exponentially stable equilibrium, $\gamma=\kappa, \mathcal{L}_{p}=\mathcal{H}_{p}=\mathcal{N}_{p}$, and $\xi=\alpha$ can be attained. In the case they are attained, $\tau=\frac{\log \max \left(\mu, \frac{\xi}{\kappa}\right)}{\varepsilon}=\frac{\log \mu}{\varepsilon}$, (35) collapses to (7), and we can consider this a true generalization of the result in [9]. In fact, one can notice that both examples in [9] satisfy the equality $\mu \kappa=\xi$.

Remark 4. Using similar techniques as those in Theorem 4, one can find a bound for $\tau$ and $\mathcal{M}$ that looks like $\min \left(\max \left(\mu \gamma, \eta_{p}\right), \xi_{p}\right)$. This expression reduces to $\xi_{p}$ given Proposition 5.

Remark 5. We chose to define the dwell time as a constant across all subsystems, but allowed the spatial calculation to proceed with regard to individual subsystems. This is consistent with the idea that applications will utilize a single, subsystem invariant, dwell time. 


\section{Examples}

We provide two numerical examples to illustrate the main results of the paper. In particular, we want to demonstrate that tight tailoring can provide better results than expanding the entry neighborhood.

\subsection{One-Dimensional Example}

Consider a family of systems defined by

$$
f_{1}(x)=-x(x-1)^{2}, f_{2}(x)=-(x-1) .
$$

Select $V_{1}(x)=x^{2}$ and $V_{2}(x)=(x-1)^{2}$ and $\delta=[0.05 ; 0.1]$. Then, $\mathcal{H}_{1}=[-0.139,0.197] \cup[0.803,1.139], \mathcal{H}_{2}=[0.776,1.224], \mu=67.28$, and $\varepsilon=$ 1.29. We can set $\boldsymbol{\kappa}=[0.0193 ; 0.050]$, giving $\mathcal{N}_{1}=[-0.139,0.139] \subset \mathcal{H}_{1}$ and $\mathcal{N}_{2}=[0.776,1.224] \subset \mathcal{H}_{2}$. Then, we can compute $\boldsymbol{\gamma}=[1.297 ; 0.050], \mathcal{L}_{1}=$ $[-1.139,1.139], \mathcal{L}_{2}=[0.776,1.224]$, and $\boldsymbol{\xi}=[1.497 ; 4.575]$, where $\boldsymbol{\gamma}=\left\{\gamma_{p}\right\}$ and $\boldsymbol{\xi}=\left\{\xi_{p}\right\}$. Finally, this results in $\tau=4.240$ and $\mathcal{M}=[-1.224,3.139]$. The size of $\mathcal{M}$ is 4.362 .

We compare the large- $\kappa$ method with two metrics. First, we select $\kappa$ so that $\tau$ is the same as the tight-tailoring method (we make the methods temporally-equivalent) and compare the size of $\mathcal{M}$. Second, we select $\kappa$ so that the size of $\mathcal{M}$ is the same as the tight-tailoring method (we make the methods spatially-equivalent) and compare the required dwell time. In order to make $\tau=4.240$, we must set $\kappa=[2.271 ; 0.257]$. This results in $\mu=8.837$, $\varepsilon=0.514$, and $\mathcal{M}=[-1.507,3.507]$, which has size 5.014. For the second case, we can make $\mathcal{M}=[-1.181,3.181]$ by setting $\kappa=[1.395 ; 0.0328]$. The result is that $\mu=42.48, \varepsilon=0.0657$, and $\tau=57.08$. In each case, tight tailoring shows better performance.

\subsection{Two-Dimensional Limit Cycle Example}

Consider a family of systems defined by

$$
\begin{gathered}
\mathbf{f}_{1}\left(\begin{array}{l}
x \\
y
\end{array}\right)=\left[\begin{array}{cc}
-\left(x^{2}+y^{2}-1\right) & -1 \\
1 & -\left(x^{2}+y^{2}-1\right)
\end{array}\right]\left(\begin{array}{l}
x \\
y
\end{array}\right) \\
\mathbf{f}_{2}\left(\begin{array}{l}
x \\
y
\end{array}\right)=\left[\begin{array}{cc}
-\left(x^{2}+y^{2}+1\right) & -1 \\
1 & -\left(x^{2}+y^{2}+1\right)
\end{array}\right]\left(\begin{array}{l}
x \\
y
\end{array}\right) \\
\mathbf{f}_{3}\left(\begin{array}{l}
x \\
y
\end{array}\right)=\left[\begin{array}{cc}
-1 & -1 \\
1 & -1
\end{array}\right]\left(\begin{array}{l}
x \\
y
\end{array}\right)+\left(\begin{array}{c}
2 \\
-2
\end{array}\right) .
\end{gathered}
$$



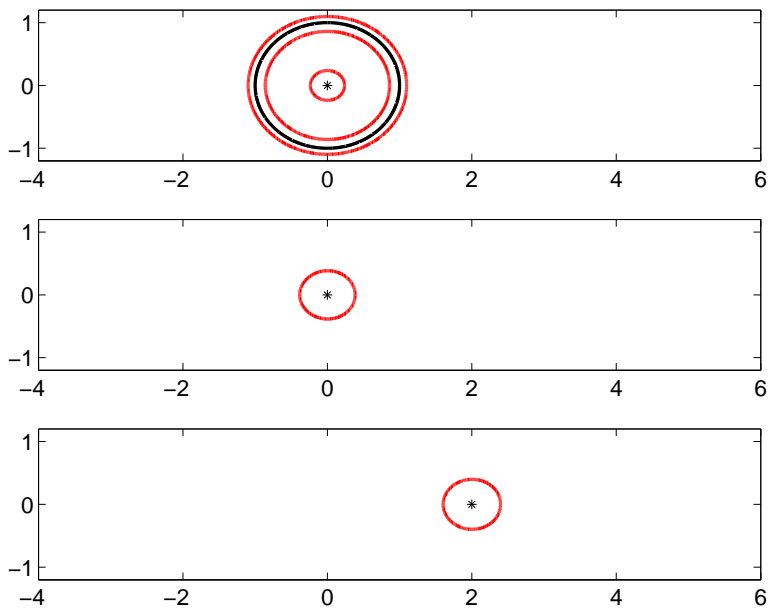

Figure 5: The sets $E_{p}$ (black) and $\partial \overline{\mathcal{H}}_{p}$ (red) for each subsystem.
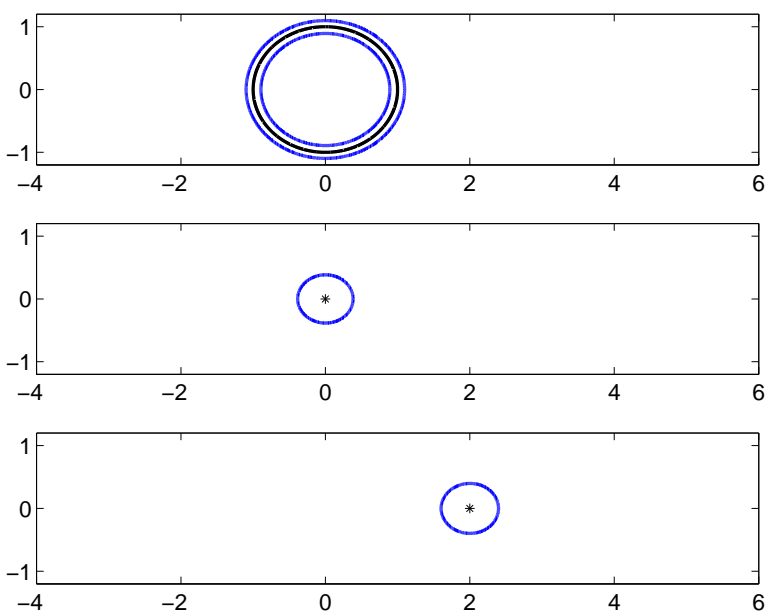

Figure 6: The sets $\mathcal{G}_{p}$ (black) and $\partial \mathcal{N}_{p}$ (blue) for each subsystem. The example is suitably well-behaved so that $E_{p}=\mathcal{G}_{p}$ and $\mathcal{H}_{p}=\mathcal{N}_{p}$ except for the unstable origin and its surrounding neighborhood when $p=1$. 


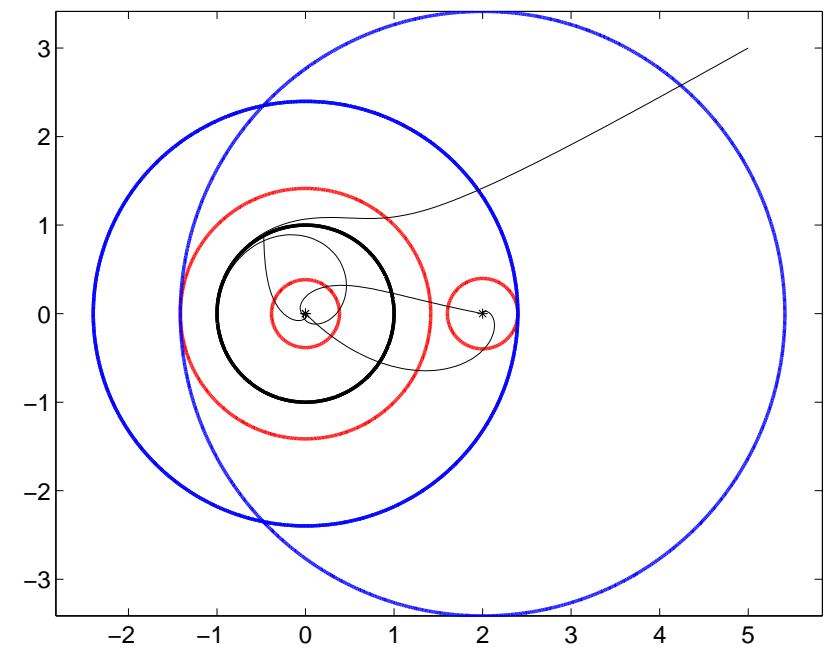

Figure 7: The sets $E_{p}$ (black), $\partial \mathcal{L}_{p}$ (red), and $\partial \mathcal{M}_{p}$ (blue). The skinny black line is an example trajectory.

This is a simplified version of (1), a central pattern generator used for control of swimming [12] or flying robots [2]. Subsystem 1 has an unstable equilibrium at the origin and a stable limit cycle of unit radius centered around the origin. Subsystem 2 has a stable equilibrium at the origin. Subsystem 3 has a stable equilibrium at $\left[\begin{array}{ll}2 & 0\end{array}\right]^{T}$. Take the corresponding Lyapunov functions to be $V_{1}(\mathbf{x})=\left(x^{2}+y^{2}-1\right)^{2}, V_{2}(\mathbf{x})=\left(x^{2}+y^{2}\right)^{2}$, and $V_{3}(\mathbf{x})=\left((x-2)^{2}+y^{2}\right)^{2}$, respectively. If we choose $\boldsymbol{\delta}=[0.2,0.1,0.1]$, then $\epsilon=0.22, \mu=2215$, $\boldsymbol{\kappa}=[0.042,0.022,0.025], \boldsymbol{\gamma}=[1.0,0.22,0.25], \boldsymbol{\xi}=[23,33,136]$, and $\tau=38.9$. Figures 5, 6, and 7 show the resulting sets. In fact, many of the resulting parameters (chief among them dwell time and size of $\mathcal{M}$ ) are determined through nontrivial relations. Optimization and tradeoffs between spatial performance and temporal performance are problem-specific.

\section{Conclusion}

We derived a stability result for switched systems which are constructed from subsystems which possibly contain multiple invariant sets. This amounted to a generalization and refinement of the argument presented in [9] and is in the spirit of dwell time methods for switched systems. This result can be applied to a larger class of dynamical systems than those in [9], including those which contain bifurcations or limit cycles. We provide a set of tun- 
ing parameters, $\boldsymbol{\delta}$, which cause interrelated and problem-specific effects on performance.

Future studies could be focused on determining an explicit relation between tuning parameters and performance for a subclass of systems in order to better facilitate optimization or investigating a continuous family of switched systems near a bifurcation point.

\section{Acknowledgement}

The authors would like to thank a reviewer for providing the example used in Section 3.3 and for suggestions which substantially improved the paper.

\section{Appendix A. Set Properties}

Proposition 7. Consider a family of compact, connected sets $\mathcal{N}_{p}$ indexed by a finite set $p \in \mathcal{P}$. For a particular $\mathbf{x} \in \mathcal{N}_{r}, \mathcal{Y}$ denotes the connected component of $\mathcal{N}=\bigcup_{p \in \mathcal{P}} \mathcal{N}_{p}$ that contains $\mathbf{x}$. Then,(1) $\mathbf{y} \in \partial \mathcal{Y} \rightarrow \mathbf{y} \in \partial \mathcal{N}$, and (2) $\mathbf{y} \in \partial \mathcal{Y} \rightarrow \mathbf{y} \in \partial \mathcal{N}_{q}$ for some $q$.

Proof. $\quad$ 1. Since $\mathcal{Y}$ is closed, $\mathbf{y} \in \partial \mathcal{Y} \subset \mathcal{Y} \subset \mathcal{N}$. Since $\mathcal{N}$ is also closed, it suffices to show that $\mathbf{y} \notin \operatorname{Int}(\mathcal{N})$. Proceed by contradiction. Assume $\mathbf{y} \in \operatorname{Int}(\mathcal{N})$. Then, there exists a connected neighborhood of $\mathbf{y}$ contained in $\mathcal{N}$. Since $\mathbf{y} \in \partial \mathcal{Y}$, an element of this neighborhood is not in $\mathcal{Y}$. We have reached a contradiction.

2. In some neighborhood of $\mathbf{y} \in \partial \mathcal{Y}$, there exist $\mathbf{z}_{1} \in \mathcal{Y} \subset \bigcup_{p \in \mathcal{P}} \mathcal{N}_{p}$ and $\mathbf{z}_{2} \notin \mathcal{Y}$. Clearly, there exists some $q$ for which $\mathbf{z}_{1} \in \mathcal{N}_{q}$. Since $\mathcal{N}_{q}$ is connected, $\mathcal{N}_{q} \subset \mathcal{Y}$. Therefore, $\mathbf{z}_{2} \notin \mathcal{N}_{q}$.

Proposition 8. For $\mathbf{x} \in \mathbb{R}^{n}$ and continuous, real-valued $f, \mathcal{X}=\{\mathbf{x}: f(\mathbf{x}) \leq$ $c\} \rightarrow \partial \mathcal{X} \subset\{\mathbf{x}: f(\mathbf{x})=c\}$.

Proof. Consider $\mathbf{y} \in \partial \mathcal{X}$. Since $f$ is continuous at $\mathbf{y}, \forall \varepsilon>0, \exists \delta>0$ s.t. $\|\mathbf{y}-\mathbf{z}\|<\delta \rightarrow|f(\mathbf{y})-f(\mathbf{z})|<\varepsilon$. Since $\|\mathbf{y}-\mathbf{z}\|<\delta$ describes a neighborhood of $\mathbf{y}$ and $\mathbf{y} \in \partial \mathcal{X}$, there exist $\mathbf{z}_{1}, \mathbf{z}_{2}$ in this neighborhood such that $\mathbf{z}_{1} \in$ $\mathcal{X}, \mathbf{z}_{2} \notin \mathcal{X}$. From $\left|f(\mathbf{y})-f\left(\mathbf{z}_{1}\right)\right|<\varepsilon$, we have

$$
f(\mathbf{y})<f\left(\mathbf{z}_{1}\right)+\varepsilon<c+\varepsilon .
$$


From $\left|f(\mathbf{y})-f\left(\mathbf{z}_{2}\right)\right|<\varepsilon$, we have

$$
f(\mathbf{y})>f\left(\mathbf{z}_{2}\right)-\varepsilon>c-\varepsilon .
$$

Taking $\varepsilon$ arbitrarily small implies that $f(\mathbf{y})=c$.

[1] G. Chen, J. L. Moiola, H. O. Wang, Bifurcation control: theories, methods, and applications, I.J. Bifurcation and Chaos 10 (3) (2000) 511-548.

[2] S.-J. Chung, M. Dorothy, Neurobiologically inspired control of engineered flapping flight, Journal of Guidance, Control, and Dynamics 33 (2) (2010) 440-453.

[3] D. Liberzon, Switching in Systems and Control, Birkhäuser Boston, 2003.

[4] J. P. Hespanha, D. Liberzon, D. Angeli, E. D. Sontag, Nonlinear normobservability notions and stability of switched systems, IEEE Trans. on Auto. Cont. 50 (2005) 154-168.

[5] A. Bacciotti, L. Mazzi, An invariance principle for nonlinear switched systems, Systems \& Control Letters 54 (2005) 1109-1119.

[6] J. L. Mancilla-Aguilar, R. Garcia, An extension of LaSalle's invariance principle for switched systems, Systems \& Control Letters 55 (2005) 376-384.

[7] S. Mastellone, D. M. Stipanović, M. W. Spong, Stability and convergence for systems with switching equilibria, in: Proc. of the 46th IEEE Conference on Decision and Control, 2007.

[8] X. Xu, G. Zhai, S. He, On practical asymptotic stabilizability of switched affine systems, Nonlinear Analysis: Hybrid Systems 2 (1) (2008) 196208.

[9] T. Alpcan, T. Başar, A stability result for switched systems with multiple equilibria, Dynamics of Continuous, Discrete and Impulsive Systems Series A: Mathematical Analysis 17 (2010) 949-958.

[10] J. LaSalle, Some extensions of Liapunov's second method, IRE Transactions on Circuit Theory 7 (4) (1960) 520-527. 
[11] N. P. Bhatia, On asymptotic stability in dynamical systems, Mathematical Systems Theory 1 (2) (1967) 113-127.

[12] K. Seo, S.-J. Chung, J.-J. E. Slotine, CPG-based control of a turtle-like underwater vehicle, Autonomous Robots 28 (3) (2010) 247-269. 